\title{
Deciphering flux adjustments of engineered $E$. coli cells during fermentation with changing growth conditions
}

\author{
Lian $\mathrm{He}^{1^{*}}, \mathrm{Yu} \mathrm{Xiu}^{2,3^{*}}$, J. Andrew Jones ${ }^{2,4^{*}}$, Edward E. K. Baidoo ${ }^{5}$, Jay D. Keasling ${ }^{5-10}$, Yinjie J. Tang ${ }^{1 \dagger}$, \\ Mattheos A. G. Koffas ${ }^{2 \dagger}$ \\ ${ }^{1}$ Department of Energy, Environmental, and Chemical Engineering, Washington University, St. Louis, \\ MO, USA \\ ${ }^{2}$ Department of Chemical Engineering, Center for Biotechnology and Interdisciplinary Studies, \\ Rensselaer Polytechnic Institute, Troy, NY, USA \\ ${ }^{3}$ College of Life Science and Technology, Beijing University of Chemical Technology, Beijing, China \\ ${ }^{4}$ Department of Chemistry, Hamilton College, Clinton, NY, USA \\ ${ }^{5}$ Joint BioEnergy Institute, Emeryville, CA, USA \\ ${ }^{6}$ Department of Chemical \& Biomolecular Engineering, University of California, Berkeley, CA, USA \\ ${ }^{7}$ Department of Bioengineering, University of California, Berkeley, CA, USA \\ ${ }^{8}$ Biological Systems and Engineering Division, Lawrence Berkeley National Laboratory, Berkeley, CA, \\ USA
}

${ }^{9}$ California Institute of Quantitative Biosciences (QB3), University of California, Berkeley, CA, USA

${ }^{10}$ Novo Nordisk Foundation Center for Biosustainability, Technical University of Denmark, Kogle Allé, DK2970-Hørsholm, Denmark

*: Equal contributions

$\uparrow:$ Correspondents

YT: yinjie.tang@wustl.edu

MK: koffam@rpi.edu 


\section{Abstract}

Microbial fermentation conditions are dynamic, due to transcriptional induction, nutrient consumption, or changes to incubation conditions. In this study, ${ }^{13} \mathrm{C}$-metabolic flux analysis was used to characterize two violacein-producing E. coli strains with vastly different productivities, and to profile their metabolic adjustments resulting from external perturbations during fermentation. The two strains were first grown at $37^{\circ} \mathrm{C}$ in stage 1 , and then the temperature was transitioned to $20^{\circ} \mathrm{C}$ in stage 2 for the optimal expression of the violacein synthesis pathway. After induction, violacein production was minimal in stage 3, but accelerated in stage 4 (early production phase) and 5 (late production phase) in the high producing strain, reaching a final concentration of $1.5 \mathrm{mmol} / \mathrm{L}$. On the contrary, $\sim 0.02 \mathrm{mmol} / \mathrm{L}$ of violacein was obtained from the low producing strain. To have a snapshot of the temporal metabolic changes in each stage, we performed ${ }^{13} \mathrm{C}-\mathrm{MFA}$ via isotopomer analysis of fast-turnover free metabolites. The results indicate strikingly stable flux ratios in the central metabolism throughout the early growth stages. In the late stages, however, the high producer rewired its flux distribution significantly, which featured an upregulated pentose phosphate pathway and TCA cycle, reflux from acetate utilization, negligible anabolic fluxes, and elevated maintenance loss, to compensate for nutrient depletion and drainage of some building blocks due to violacein overproduction. The low producer with stronger promoters shifted its relative fluxes in stage 5 by enhancing the flux through the TCA cycle and acetate overflow, while exhibiting a reduced biomass growth and a minimal flux towards violacein synthesis. Interestingly, the addition of the violacein precursor (tryptophan) in the medium inhibited high producer but enhanced low producer's productivity, leading to hypotheses of unknown pathway regulations (such as metabolite channeling).

Key words: ${ }^{13} \mathrm{C}-\mathrm{MFA}$; channeling; free metabolites; promoter; reflux; tryptophan; violacein 


\section{Introduction}

Cell metabolism involves coordination of cellular processes at multiple levels (Xu et al., 2012a; Cress et al., 2015; Wu et al., 2016). This complexity of biological systems is always challenging to understand, yet can be addressed by studying metabolic adaptations towards environmental and genetic perturbations, which requires multiple omics knowledge and/or computational simulations (Buescher et al., 2012; Facchetti, 2016; Jones et al., 2015a; van Heerden et al., 2014). Among those techniques facilitating cellular metabolism analysis, ${ }^{13} \mathrm{C}$ metabolic flux analysis (MFA) is the only method to quantify the in vivo enzymatic reaction rates in a network via combining isotopomer tracer experiments and computational efforts. The resulting flux distributions reflect the functional outputs of gene-protein-metabolite interactions (Sauer, 2006). ${ }^{13} \mathrm{C}-\mathrm{MFA}$ is particularly useful to examine metabolic responses to genetic and environmental alternations (Fischer and Sauer, 2005). To date, there have been many research studies either characterizing metabolic flux adaptations towards diverse growth conditions (Fischer and Sauer, 2003; Kayser et al., 2005; Rühl et al., 2012) or profiling the flux reorganizations to support bioproduction in engineered hosts (Kind et al., 2013; Xiong et al., 2015). Most of these flux analyses rely on isotopomer analysis of proteinogenic amino acids synthesized under steady-state conditions (Wittmann, 2007; Zamboni et al., 2009). To extend the capability of ${ }^{13} \mathrm{C}$ MFA and to resolve the flux oscillation in growth transitions or under successive perturbations, dynamic flux analysis has been developed (Antoniewicz, 2013; Leighty and Antoniewicz, 2011). This technique has been applied to decipher the metabolic heterogeneity in the presence of microbial subpopulations by tracking the labeling dynamics of fast-turnover free metabolites (van Heerden et al., 2014). 
In this study, we focused on two engineered violacein-producing $E$. coli strains with vastly different violacein productivities and investigated changes of their fluxomes throughout an entire cultivation period. Violacein is a natural product with a vibrant purple color and interesting antibacterial activity (McClean et al., 1997). In our previous work, we have successfully improved the violacein titer by over 450 folds via optimization of growth conditions, time of gene expression, and transcriptional strengths (Jones et al., 2015b). Interestingly, the mutant strain with the highest violacein production expresses the violacein biosynthetic pathway from very weak promoters. To understand the influence of gene expression strength on flux distributions, we selected a high producer (stain E12) and a low producer (strain Consensus) for analysis (Figure 1). During cultivation, both engineered E. coli strains were subjected to a temperature reduction from $37^{\circ} \mathrm{C}$ to $20^{\circ} \mathrm{C}$ and transcriptional induction with IPTG (Figure $2 \mathrm{~A}$ B). Instead of using proteinogenic amino acids, we measured the labeling patterns of free metabolites, which has been shown to be a reliable approach of capturing metabolic fluxomes (Toya et al., 2007). By using free metabolites and quantifying the alterations of metabolic flux phenotypes, we have developed a method to better understand the metabolic adaptations resulting from successive perturbations of fermentation conditions in engineered hosts. Profiling fluxes in a continuous growth period will allow a quantitative understanding of cell metabolic regulations that may shed light on the metabolic tradeoff between robustness and adaptability for bio-production. This information also provides useful guidelines for rational metabolic engineering. Furthermore, gene expression analysis has been frequently performed in microbial physiological studies to, for example, understand the correlation of genetic expressions to phenotypic variations; however, genome-to-phenome mapping is not straightforward and the 
causation of metabolic responses is still poorly understood. Hence, this study can offer insights into phenotypic heterogeneity between the two strains with near-identical genetic backgrounds.

\section{Materials and method}

\subsection{Bacterial cultivation and ${ }^{13} \mathrm{C}$ labeling experiment}

Two engineered violacein-producing E. coli strains (Jones et al., 2015b) were evaluated: strain E12 (the high producer) and strain Consensus (the low producer). Both strains contain a single plasmid, constructed in monocistronic form with a separate promoter and terminator controlling the transcription of each gene in the 5-gene pathway, using the ePathBrick method (Xu et al., 2012b; Whitaker et al., 2016). The two plasmids varied only by the identity of the mutant T7 promoters controlling the transcription of the violacein pathway. These changes amounted to a difference in 17 base pairs spread over the five promoter regions (Figure 1A). BL21 $\mathrm{Star}^{\mathrm{TM}}$ (DE3) was used as the host strain for expression of both plasmids. To prepare seed cultures, single colonies were inoculated into Andrew's Magic Media (AMM) (He et al., 2015; Zhao et al., 2015) without casamino acids, containing $15 \mathrm{~g} / \mathrm{L}\left[1,2-{ }^{13} \mathrm{C}_{2}\right]$ glucose (Sigma-Aldrich, MO, USA). This seed culture was grown overnight at $37^{\circ} \mathrm{C}, 225 \mathrm{rpm}$, and then inoculated $(2 \% \mathrm{v} / \mathrm{v})$ into the same fresh media as above. The labeled cultures were first grown at $37^{\circ} \mathrm{C}$ for 5.5 hours till $\mathrm{OD}_{600}$ was $\sim 1.2$, and then moved to a $20^{\circ} \mathrm{C}$ growth chamber. At $20^{\circ} \mathrm{C}$, they were grown for one hour before 1mM IPTG induction. Cell cultures were sampled at five different time points: 1) immediately before the temperature change from $37^{\circ} \mathrm{C}$ to $20^{\circ} \mathrm{C} ; 2$ ) immediately before IPTG induction at $20^{\circ} \mathrm{C}$; 3) two hours after IPTG induction; 4) six hours after IPTG induction; and 5) ten hours after IPTG induction (Figure 2B). We observed that the cells required one hour to adapt to a particular change in their environment (either temperature or induction); hence, we allowed the cells to adapt to the new environment before taking samples for flux analysis. We grew multiple labeled 
cultures (15 ml cultures in $125 \mathrm{ml}$ shake flasks, Figure $2 \mathrm{~A})$ simultaneously. At each time point, a whole culture was harvested for each engineered strain. Specifically, $4 \mathrm{ml}$ of a labeled culture was used for analyzing the labeling patterns of proteinogenic amino acids, $10 \mathrm{ml}$ for analyzing free metabolites, and finally $1 \mathrm{ml}$ for glucose, acetate, and violacein concentration measurements.

\subsection{Cell viability assay}

To evaluate the cell viability at the late stage of fermentation (Figure 2B), the two strains were cultivated in unlabeled AMM media and stained by using LIVE/DEAD ${ }^{\circledR}$ BacLight $^{\mathrm{TM}}$ Bacterial Viability Kits (L7012, Molecular Probes). With the excitation wavelength centered at 485nm, the fluorescence intensity of stained bacterial suspensions was measured at 530nm (emission 1, green) and 630nm (emission 2, red) separately by fluorescence microplate readers. The live cell percentage was determined by dividing the fluorescence intensity of the stained bacterial suspensions at emission 1 by the corresponding fluorescence intensity at emission 2 . The resulting ratios were then quantified against a standard Live/Dead curve per manufacturer's instructions.

\subsection{Analytical methods}

To analyze proteinogenic amino acids, the biomass samples were first treated with $6 \mathrm{M} \mathrm{HCl}$ at $100^{\circ} \mathrm{C}$ for 24 hours. The resulting mixtures were centrifuged to remove undissolved impurities, and then the supernatant was air-dried. Tetrahydrofuran and $N$-tert-butyldimethylsilyl- $N$ methyltrifluoroacetamide (TBDMS) were added into dried samples at a ratio of $1: 1(\mathrm{v} / \mathrm{v})$ to

derivatized proteinogenic amino acids prior to GC/MS (Hewlett-Packard model 7890A, Agilent Technologies) analysis (You et al., 2014). 
To prepare samples for LC/MS analysis (Hollinshead et al., 2016), labeled cultures from the incubator were quickly poured into pre-frozen centrifuge tubes that were bathed in liquid nitrogen. Then the culture was vigorously stirred for several seconds to freeze cells near $0^{\circ} \mathrm{C}$. The quenched samples were centrifuged for 2 minutes at $0^{\circ} \mathrm{C}$, and the supernatant was discarded. To extract the free metabolites from quenched biomass, methanol and chloroform (7:3) were added to the biomass samples. The mixtures were shaken at $200 \mathrm{rpm}$ at $4^{\circ} \mathrm{C}$ for 4 hours, and then centrifuged at $9000 \mathrm{rpm}$ for 10 minutes. The top layer containing free metabolites was transferred into ultra- $0.5 \mathrm{~mL}$ centrifugal filters (EMD Millipore, Germany), and then were centrifuged at $9000 \mathrm{rpm}$ to remove macromolecules. The samples were lyophilized and stored at $80^{\circ} \mathrm{C}$ before LC/MS analysis. The labeling patterns of free metabolites or proteinogenic amino acids were determined by mass spectrometry data. Here, we used $\mathrm{M}+0, \mathrm{M}+1, \mathrm{M}+2$, etc., to represent relative abundances of non-labeled, singly-labeled, and doubly-labeled metabolites, respectively.

HPLC analysis was performed to measure concentrations of violacein, glucose, and acetate (Jones et al., 2015b; Whitaker W. B. et al., 2016). Violacein, acetate, and biomass yields were determined by linear correlations between product and glucose concentrations, and the yields were used to loosely constrain ${ }^{13} \mathrm{C}$ MFA models. Due to the absorbance of violacein at $600 \mathrm{~nm}$, measurements of $\mathrm{OD}_{600}$ in this manuscript should be interpreted as a linked measure of cell growth and violacein production. Besides, violacein may precipitate at high concentrations and interfere with $\mathrm{OD}_{600}$ analysis (Sun et al., 2016). So the $\mathrm{OD}_{600}$ data in the violacein production phase were not used for biomass estimations. 


\section{4. ${ }^{13} \mathrm{C}$ metabolic flux analysis for a continuous growth period}

The central metabolic network was modified from our previous study (He et al., 2014) by including two violacein biosynthesis equations: ' 2 tryptophan $+2 \mathrm{NADPH} \rightarrow$ violacein $+2 \mathrm{CO}_{2}$ ', and '2 tryptophan $+\mathrm{NADPH} \rightarrow$ deoxyviolacein $+2 \mathrm{CO}_{2}$ ' (see Table S1). To quantify the fluxes through the central metabolism, cell growth data (Table S2) and the labeling patterns of eleven free metabolites (i.e., ALA, ASP, FBP, G6P, GLU, LEU, MAL, MET, PHE, Ru5P, and SUC, see Table S3) were used. The following assumptions were applied before flux calculations: 1) the enzymes in violacein biosynthesis accounted for only a small part of the overall protein content, and the biomass compositions (i.e., biomass equation) did not undergo significant changes in either low- or high-producers; 2) at each time point, labeled cultures were under a pseudo-steady-state condition, since their growth condition was not subject to any sudden perturbation; and 3) ${ }^{13} \mathrm{C}$ purity was $99 \%$ for $\left[1,2-{ }^{13} \mathrm{C}\right]$ glucose and the unlabeled carbons had a natural ${ }^{13} \mathrm{C}$ abundance of $1.07 \%$ (Nanchen et al., 2007). The elementary metabolite unit algorithm was used to simulate the labeling patterns of free metabolites (Antoniewicz et al., 2007), and flux calculations was performed by using MATLAB ${ }^{\circledR} 2016$ b. Fifty initial guesses were tested to find the global optimum that gives the least differences between experimentally measured and computationally simulated data. To calculate the $95 \%$ confidence intervals (CIs) of flux variables, we perturbed the experimental data with normally distributed noises $(\mu=0$, $\delta=0.02)$ and recalculated fluxes 200 times. The CIs were determined by the top and bottom $2.5 \%$ values, and the standard deviations were calculated by using the following equation: (Upper 95\% CI bound - Lower 95\% CI bound)/3.92 (Young et al., 2011). 


\subsection{SDS/PAGE of tryptophan feeding experiment}

The two strains E12 and Consensus were cultivated in unlabeled AMM media as described in 2.1, except that $50 \mathrm{mg} / \mathrm{L}$ tryptophan was added into cultures at $t_{3}$ or $t_{4}$ after IPTG induction. The whole culture was harvested after fermentation ( $t_{5}$, Figure $\left.2 \mathrm{~B}\right)$ for HPLC analysis of violacein titers and SDS/PAGE analysis of protein expressions. Cell pellets were resuspended in Tris buffer $(50 \mathrm{mM}$ Tris $\cdot \mathrm{HCl}, 150 \mathrm{mM} \mathrm{NaCl}$, and $\mathrm{pH} 7.5)$ and then sonicated $(60 \%$ output, 5 sec pulse and $5 \mathrm{sec}$ rest for $3 \mathrm{~min}$ ) to release the intracellular soluble proteins. Total protein concentration was measured with BCA protein assay. Under different conditions of IPTG induction and tryptophan addition, the same amount of total protein (15ng) of each sample was loaded on SDS/PAGE. Gel was stained with SimpleBlue ${ }^{\mathrm{TM}}$ SafeStain for an hour and destained with deionized water overnight.

\section{Results}

\subsection{Labeling experiment and physiology of violacein-producing $E$. coli strains}

In the ${ }^{13} \mathrm{C}$ labeling experiment, we grew strain $\mathrm{E} 12$ and Consensus in a defined medium containing $15 \mathrm{~g} / \mathrm{L}\left[1,2-{ }^{13} \mathrm{C}_{2}\right]$ glucose. To ensure a high violacein yield, the cultures were first grown at $37^{\circ} \mathrm{C}$ for biomass growth, and then the temperature was switched to $20^{\circ} \mathrm{C}$ for optimal pathway expression and product synthesis (Figure 2A) (Jones et al,, 2015b). The lowtemperature cultivation was also beneficial for cell survival under violacein stresses. Samples of both proteinogenic amino acids and free metabolites were harvested at the five growth stages illustrated in Figure 2B.

The physiological performance of both engineered strains in the ${ }^{13} \mathrm{C}$ labeling experiment is shown in Figure $2 \mathrm{C}$ and Table $\mathrm{S} 2$. From $t_{2}$ to $t_{4}$, the acetate yield in strain Consensus was 
negligible and less than $0.01 \mathrm{mmol}$ acetate $/ \mathrm{mmol}$ glucose, however, this value spiked to over 0.6 mmol acetate $/ \mathrm{mmol}$ glucose at $t_{5}$. Strain E12 appears an opposite trend: it produced $\sim 0.2 \mathrm{mmol}$ acetate $/ \mathrm{mmol}$ glucose from $t_{1}$ to $t_{4}$, but started consuming acetate at $t_{5}$. With respect to violacein production, strain E12 produced $\sim 100$ times more violacein than the low-performance strain. The overall violacein yield is $\sim 0.06 \mathrm{mmol}$ violacein/ $\mathrm{mmol}$ glucose in strain E12, and negligible in strain Consensus. Although $\mathrm{OD}_{600}$ was interfered by the purple color of violacein, we didn't observe significant growth changes between the two species from $t_{1}$ to $t_{3}$ before a high level of violacein production was achieved from strain E12.

\subsection{Fast-turnover free metabolites}

Conventionally, the labeling patterns of proteinogenic amino acids are used to calculate fluxes for chemostat cultures. However, since they always contain a 'memory' of previously synthesized amino acids and their turnover rates are slow, ${ }^{13} \mathrm{C}-\mathrm{MFA}$ cannot use them to capture metabolism subject to external perturbations in a fermentation period. Instead, we measured the labeling patterns of free metabolites. The turnover rates of free metabolites are on the order of seconds (Taymaz-Nikerel et al., 2009), and thus their labeling information at a certain time point results from instant enzymatic reactions, which can be determined by LC/MS (Ma et al., 2014). In this study, ${ }^{13} \mathrm{C}-\mathrm{MFA}$ uses these fast-turnover metabolites to provide a snapshot of metabolic changes during fermentation. All the labeling data are listed in Table S3-4.

We first compare the fold changes of all free metabolite labeling data from $t_{2}$ to $t_{5}$ by using data at $t_{l}$ as the reference. On average, most of the changes are within $10 \%$ throughout the cultivation period, while more differences are observed at $t_{5}$, especially for strain E12 (Figure 3A-B, Figure S1). This phenomenon suggests a more decided flux rerouting at the final growth state than at the earlier phases, which will be addressed later. One mutual outlier, with over $10 \%$ relative change, 
in both strains is ribulose-5-phosphate (Ru5P) (Figure 3C- D). Specifically, the singly labeled fraction (i.e., $\mathrm{M}+1$ ) increased from $\sim 0.4$ during early growth phase to $\sim 0.7$ in the violaceinproducing phase. Since the non-oxidative pentose phosphate pathway is highly reversible (Follstad and Stephanopoulos, 1998), we would observe doubly, triply, and even quadruply labeled Ru5P. The significant increase in $\mathrm{M}+1$ reflects a reduced reversibility of reactions Ru5P $\leftrightarrow$ $\mathrm{R} 5 \mathrm{P}$ and Ru5P $\leftrightarrow \mathrm{X} 5 \mathrm{P}$, and thus the oxidative pentose phosphate pathway (OPPP, G6P $\rightarrow \mathrm{Ru} 5 \mathrm{P}+$ $\mathrm{NADPH}$ ) becomes a dominating source for Ru5P synthesis. This finding makes sense because more carbon fluxes from R5P are directed toward the violacein synthesis pathway at $t_{4}$ or/and $t_{5}$. In addition, OPPP flux also provides NADPH for product synthesis. Another striking change was observed in the labeling patterns of G6P and FBP at $t_{5}$ for strain E12 (Figure $3 \mathrm{E}-\mathrm{H}$ ). When glycolysis dominates central metabolism, both G6P and FBP show highest abundance (>80\%) in $\mathrm{M}+2$. Nonetheless, this value dropped to $35 \%$ and $53 \%$ for FBP and G6P, respectively. Meanwhile, $\mathrm{M}+1$ abundances in both metabolites increased by over 10 folds. The explanation for this phenomenon is that strain E12 has a higher relative flux through OPPP, so that more ${ }^{13} \mathrm{C}$ labeled carbons are lost as $\mathrm{CO}_{2}$ and more singly labeled metabolites are generated.

Although there are labeling differences caused by non-steady-state conditions, both proteinogenic and free amino acids as well as the two engineered strains should have the same labelling patterns at $t_{l}$ when the growth condition has not yet been changed. As expected, they show strong linear correlations (Figure S2 A), confirming a pseudo-steady-state cell metabolism. We further compared fluxes determined by proteinogenic amino acids and free metabolites at $t_{l}$. The flux estimations resulting from those two sets of data are in accordance with each other (Figure S2 B-C), indicating that either free metabolites or proteinogenic amino acids can be applied for ${ }^{13} \mathrm{C}-\mathrm{MFA}$ on exponentially growing cells. Moreover, ${ }^{13} \mathrm{C}-\mathrm{MFA}$ indicated a poor 
fitting to 3-phosphoglycerate (3PG) labeling patterns in both engineered strains. Coincidentally, a previous study also reported the same issue that arguably resulted from inactive metabolite pools (Toya et al., 2007). For example, glycolysis has been shown to be channeled and thus the intermediate metabolites outside the pathway channel form metabolic inactive pools with sluggish turnover rates (Shearer et al., 2005). The presence of metabolically inactive pools may dilute the overall labeling patterns, however, no extraction method can distinguish between active and inactive metabolite pools. Therefore, we did not use the labeling patterns of 3PG for investigating the flux phenotypes.

\subsection{Central metabolic responses to genetic differences and environmental changes.}

After exposure to a sudden environment perturbation, cells should undergo flux oscillations and adjustments (van Heerden et al., 2014). Here, we aimed to characterize cells' flux phenotypes when they reached steady states at different stages. Therefore, the conventional ${ }^{13} \mathrm{C}$ MFA algorithm for steady-state metabolism was employed. The resulting flux distributions at the five time points are shown in Table S5-6, and the fittings to measured data are presented in Figure S3.

To gain a general picture of flux rerouting throughout the cultivation time, we use a heat map to present the relative flux changes from $t_{2}$ to $t_{5}$ compared to those at $t_{1}$ (Figure 4A). Intriguingly, the flux topology in both strains remained robust against temperature change and IPTG induction, which is evidenced by the fact that most of the relative changes are within $30 \%$ from $t_{1}$ to $t_{4}$ (Figure S4). The steady labeling patterns of proteinogenic amino acids from time-course biomass samples also supports the robustness of relative fluxes in central metabolisms during cell growth (Table S4). Particularly, the relative fluxes from glucose 6-phosphate (G6P) to the glycolysis and OPPP appear to be the most rigid, even though the acetate yield varies temporally. As shown in Figure $4 \mathrm{~B}$, during the entire growth period, $83 \pm 3 \%$ of carbon flux from glucose uptake is 
directed towards glycolysis pathway and $15 \pm 4 \%$ towards the OPPP in strain Consensus. This rigidity also applies to strain E12 from $t_{1}$ to $t_{3}$ with similar flux partitions towards glycolysis and the OPPP. Fluxes through the TCA cycle and anaplerotic pathways demonstrated more changes and thus more flexibility, which is partly due to varying acetate yields throughout the cultivation. As a consequence of the rigidity, the total relative flux of the central carbon metabolism remains conserved. By summing up all the relative fluxes in the central metabolism, we found that this value barely changed $(13 \pm 0.8$ times of the glucose uptake rate, Figure $4 \mathrm{C})$ before $t_{5}$. Taken together, E. coli cells show a strong preference for relative stable flux topology to allocate carbon fluxes among central pathways even under environmental and genetic stresses (i.e., metabolic robustness). Such stability is most likely favorable to their survival and proliferation capacity.

Although E. coli cells are robust in flux topology, flux rerouting can be triggered by either violacein accumulation or nutrient insufficiency at $t_{5}$. As a result, both high and low producers show different flux phenotypes from the previous ones (Figure 4A and D). Strain Consensus features a highly elevated flux through the TCA cycle at $t_{5}$, which mimics the flux phenotype of E. coli growing on limited glucose in chemostat bioreactors (Kayser et al., 2005). Considering a surge of acetate overflow at this state, it is likely that strain Consensus is under such an energylimited condition that biomass synthesis is largely sacrificed to support a higher energy generation from glucose (Figure 4D). To calculate the flux distribution of strain E12 at $t_{5}$, we included the acetate uptake reaction due to the observation of reduced acetate concentration from $t_{4}$ to $t_{5}$ (Figure $2 \mathrm{C}$ ). This diauxic growth on both glucose and acetate indicates limiting carbon, which is representative of stationary growth phase. Since acetate/acetyl-CoA is mainly generated from pyruvate, the major precursor to alanine, we used the labeling information of Ala $[\mathrm{M}-85]^{+}$ 
to infer the labeling patterns of acetate and fractions of different isotopologues (i.e., molecules differing only in isotopic compositions) present in the medium (Table S4). As revealed by flux calculations, strain E12 exhibits progressively elevated relative fluxes through the OPPP from $t_{3}$ to $t_{5}$, which ensures sufficient production of erythrose 4-phosphate (and NADPH) for tryptophan and violacein synthesis. The other two precursors, serine and phosphoenolpyruvate, can be provided through glycolysis. Due to the acetate consumption at $t_{5}$, the relative flux of the TCA cycle is highly enhanced, together with an increased gluconeogenic flux through the PEP carboxykinase reaction. Strikingly, there is barely any net biomass growth of strain E12 at $t_{5}$, suggesting that most carbon resources are used for energy production rather than violacein and biomass synthesis.

Finally, we want to point out that the fitting quality of labeled free metabolites significantly decreased at $t_{5}$ for strain E12, especially for G6P and FBP. One reason would be the existence of subpopulations with different flux phenotypes (Xiao et al., 2016). Due to product toxicity, cell death rate may increase as evidenced by the cell viability test showing that significant dead cells were present in strain E12 cultures at the late stages. In contrast, Consensus strain has much higher cell survival ratio (Table S7). Such a metabolic heterogeneity can be quantitatively deciphered by using reporter proteins or peptides that are unique to different subpopulations (Ghosh et al., 2014; Rühl et al., 2011). A computational algorithm for ${ }^{13} \mathrm{C}-\mathrm{MFA}$ of subpopulations has also been proposed (Gebreselassie and Antoniewicz, 2015). However, these techniques cannot be directly applied in this study.

\subsection{Evaluating the metabolic bottleneck for violacein production}

${ }^{13} \mathrm{C}$-MFA studies have inspired engineering approaches for bio-production enhancement (Kind et al., 2013). Therefore, we attempted to use ${ }^{13} \mathrm{C}-\mathrm{MFA}$ to investigate why strain Consensus with 
stronger promoters produced less violacein than strain E12 with weaker promoters. The first hypothesis is that expressing heterologous pathways in strain Consensus drains cellular resources, thereby causing fluxome reorganization and metabolic burdens (Wu et al., 2016). However, a comparison of fluxomes between the two strains from stage 1 to 3 does not support this hypothesis. Furthermore, we supplied additional $50 \mathrm{mg} / \mathrm{L}$ tryptophan into the medium at the late fermentation stages to improve the intracellular tryptophan level and increase violacein production. As a result, strain Consensus showed a $40 \%$ increase in violacein production, however, this value is still much lower than strain E12 (Figure 5A). Counterintuitively, not only is violacein production decreased by $12 \%$ in strain E12, but both strains show less increase of $\mathrm{OD}_{600}$ after tryptophan addition. These observations could be partly caused by tryptophan's feedback inhibition on its own synthesis pathway (Wegman and Crawford, 1968). Considering the above results and the fact that $E$. coli can absorb exogenous tryptophan via $\mathrm{ABC}$ transporters, it is likely that the intracellular tryptophan level is not the decisive factor for the low performance of strain Consensus. These findings lead us to examine the enzymes of the violacein biosynthesis pathway. To this end, we sought to gain a qualitative comparison of protein overexpression levels between the two engineered strains by performing SDS-PAGE analysis of the soluble and insoluble proteins. In general, there is no significant overexpression of any soluble enzyme (Figure 5B) in either strain Consensus or E12. Additionally, No significant formation of inclusion bodies was seen in the insoluble protein gel (Figure S5). However, we observed slightly enhanced protein expression in the molecular weight range of enzymes, VioA (49 kDa), VioC (49 kDa), and VioD (42 kDa) in strain E12. Although the above finding does not give a compelling proof to resolve the mystery of why dramatically higher violacein titer was achieved in the strain with weaker promoters, we can deduce that: 1) the promoter strength and 
the enzyme expression can be uncorrelated, especially when multiple promoters are used to overexpress the pathway, and 2) that the overall balance of enzymatic activity of the violacein pathway is possibly not as optimal in strain Consensus as that in strain E12. Indeed, the underlying cause is certainly more complex than we initially expected, and it undoubtedly warrants additional investigation to be fully resolved.

\section{Discussion}

This study characterized the flux distributions and topologies of engineered E. coli strain with different violacein-producing capabilities during different growth regimes. The labeling patterns of intracellular free metabolites at different growth periods allow ${ }^{13} \mathrm{C}$-MFA to evaluate metabolic adaptations towards environmental (i.e., temperature and nutrient sufficiency) and genetic (i.e., IPTG induction) perturbations. Whereas both strains contain near-identical genetic backgrounds, some substantial phenotypic differences have been observed in terms of violacein production, cell vitality, and flux distributions at the late growth stages.

An interesting, but not surprising, finding is the rigidity of flux topology and accordingly conservation of total relative flux in the central carbon metabolism. Metabolic rigidity is actually a ubiquitous property, evidenced by previous reports that both bacterial and yeast cells have the propensity to maintain a rigid flux distribution against null mutations (Blank et al., 2005; Fischer and Sauer, 2005). The rigidity of relative flux distribution implies that the initial carbon source uptake rate may determine the overall enzymatic rates in cell metabolism. This property not only ensures a robust central metabolism that directly provides building blocks and energy molecules for cell proliferation, but also maintains a constant level of total intracellular metabolites to avoid a big change in osmotic pressure. The flux conservation confirms a widely accepted rule regulating cellular metabolism: cells will attempt to minimize flux adjustments despite genetic 
and environmental perturbations (Segre et al., 2002; Shlomi et al., 2005). This protection avoids costly investment on adaptations and thus favors microbial survival and evolution. It is widely believed that feedback/feedforward mechanisms, metabolic redundancy, and modularity are the main evolutionary results that confer high degrees of cellular robustness to external/environmental perturbations (Chubukov et al., 2014; Nakahigashi et al., 2009; Stelling et al., 2004).

On the other hand, the adaptability is more favored over rigidity when cells are confronted with harsher environments. How cells reorganize their flux distributions can be mutant- and substratespecific, and it is a topic that has been extensively explored in many studies. This metabolic flexibility can be attributed to many reasons. From the perspective of the metabolite-enzyme relationship, most enzymes in central metabolism are not saturated with their substrates, and therefore the fluxes are sensitive to changes at the metabolic level (Bennett et al., 2009; Park et al., 2016). Additionally, cells invest ample resources on their 'defending mechanism' in preparation for unexpected perturbations from the surroundings (Fischer and Sauer, 2005). These two tactics facilitate quick responses in the central metabolism and thus increase the possibilities of microbial survival. Undoubtedly, their adaptations towards different environments involve regulatory mechanisms at gene, transcript, and protein levels, which have been addressed in previous studies (Buescher et al., 2012; Chubukov et al., 2014; Ishii et al., 2007).

We have also noticed some limitations or potential problems in this study. First, the labeling patterns of intracellular free metabolites are more prone to measurement errors than proteinogenic amino acids, since they are of lower abundance, and labeling information could be perturbed during the sampling process. Since we focused on significant changes in relative flux distribution, errors to a certain extent can be tolerated. Other potential problems include the 
intracellular inactive metabolic pools (Ma et al., 2014), refluxes of metabolites through biomass degradation (Nargund et al., 2014), and cell subpopulations with different flux phenotypes (van Heerden et al., 2014). All these issues can reduce model fitting quality. Last but not the least is the effect of metabolite channeling on ${ }^{13} \mathrm{C}$ MFA, in which intermediates undergo consecutive multi-step enzymatic catalysis without being in equilibrium with the cytosol. Metabolite channeling has been shown to occur in almost the entire central metabolism, including glycolysis (Shearer et al., 2005), OPPP (Debnam et al., 1997), and TCA cycle (Sumegi et al., 1990). Metabolite channeling allows efficient operation of native metabolic pathways by reducing diffusion limitations. However, it can also generate secondary metabolite pools outside channeled pathways, where metabolites are much less reactive (i.e., metabolically inactive pools). Such metabolic heterogeneity may alter the labeling patterns of metabolites (such as 3PG) and thus flux calculations (Wiechert, 2001). Interestingly, the uptake of extracellular tryptophan caused inhibitions of cell growth and violacein production. This phenomenon could be explained by a channeling mechanism between tryptophan and violacein synthesis pathways (Wheeldon et al., 2016), which prevents the leakage of intermediate tryptophan outside the channeled pathways, and thus facilitates violacein production (Hollinshead et al., 2016). On the other hand, we do not rule out other hidden mechanisms that can lead to the experimental observations.

\section{Conclusion}

${ }^{13} \mathrm{C}-\mathrm{MFA}$ is an important tool to reveal metabolic regulations of engineered strains and validate synthetic biology approaches (such as promoter engineering). Our flux analysis work on the two violacein-producing $E$. coli strains during a 16-hour fermentation period has several implications. In the first place, maintaining metabolic robustness is a primary choice for $E$. coli cells under environmental stresses or enzyme overexpression, while adaptability is an alternative, but 
necessary and beneficial choice in some cases. Furthermore, the rigidity of cell metabolism can pose a challenge for strain engineers who wish to obtain a large quantity of desired products from a highly preferred built-in network. Hence, it is unwise to blindly introduce diverse heterogeneous pathways into the same microbial platform without the availability of rapid and efficient high-throughput screening technologies. Rather, choosing a suitable host having higher potentials to support the desired product(s) may save many genetic manipulations and stand a better chance of achieving high yield/titer/productivity. On the other hand, perturbation of genetic and fermentation conditions affecting the metabolic rigidity of the engineered hosts can lead to an inefficient and suboptimal metabolism, resulting in unpleasant consequences in mutant performance. Therefore, it would be beneficial to sustain cells' well-being during the metabolic engineering process. This finding supports fermentation strategies that split the microbial growth and molecule-production phases to facilitate initial, uninhibited biomass accumulation followed by efficient biomolecule production phases.

\section{Acknowledgments}

This project was funded by the National Science Foundation through grants MCB 1616619 and DBI1356669 to YJT and MCB 1448657 to MK. The LC-MS work was performed at the Joint BioEnergy Institute supported by the US Department of Energy, Office of Science, and Office of

Biological and Environmental Research, through contract DE-AC02-05CH11231 between Lawrence Berkeley National Laboratory and the US Department of Energy. 


\section{References}

Antoniewicz, M. R., 2013. Dynamic metabolic flux analysis - tools for probing transient states of metabolic networks. Curr. Opin. Biotechnol. 24, 973-978.

Antoniewicz, M. R., Kelleher, J. K., Stephanopoulos, G., 2007. Elementary metabolite units (EMU): a novel framework for modeling isotopic distributions. Metab. Eng. 9, 68-86.

Bennett, B. D., Kimball, E. H., Gao, M., Osterhout, R., Van Dien, S. J., Rabinowitz, J. D., 2009. Absolute metabolite concentrations and implied enzyme active site occupancy in Escherichia coli. Nat. Chem. Biol. 5, 593-599.

Blank, L. M., Kuepfer, L., Sauer, U., 2005. Large-scale ${ }^{13} \mathrm{C}$-flux analysis reveals mechanistic principles of metabolic network robustness to null mutations in yeast. Genome Biol. 6, R49.

Buescher, J. M., Liebermeister, W., Jules, M., Uhr, M., Muntel, J., Botella, E., Hessling, B., Kleijn, R. J., Le Chat, L., Lecointe, F., 2012. Global network reorganization during dynamic adaptations of Bacillus subtilis metabolism. Science. 335, 1099-1103.

Chubukov, V., Gerosa, L., Kochanowski, K., Sauer, U., 2014. Coordination of microbial metabolism. Nat. Rev. Microbiol. 12, 327-340.

Cress, B. F., Trantas, E. A., Ververidis, F., Linhardt, R. J., Koffas, M. A., 2015. Sensitive cells: enabling tools for static and dynamic control of microbial metabolic pathways. Curr. Opin. Biotechnol. 36, 205-214.

Debnam, P. M., Shearer, G., Blackwood, L., Kohl, D. H., 1997. Evidence for channeling of intermediates in the oxidative pentose phosphate pathway by soybean and pea nodule extracts, yeast extracts, and purified yeast enzymes. Eur. J. Biochem. 246, 283-290.

Facchetti, G., 2016. A simple strategy guides the complex metabolic regulation in Escherichia coli. Sci. Rep. 6, 27660.

Fischer, E., Sauer, U., 2003. A novel metabolic cycle catalyzes glucose oxidation and anaplerosis in hungry Escherichia coli. J. Biol. Chem. 278, 46446-46451.

Fischer, E., Sauer, U., 2005. Large-scale in vivo flux analysis shows rigidity and suboptimal performance of Bacillus subtilis metabolism. Nat. Genet. 37, 636-640.

Follstad, B. D., Stephanopoulos, G., 1998. Effect of reversible reactions on isotope label redistribution. Eur. J. Biochem. 252, 360-371.

Gebreselassie, N. A., Antoniewicz, M. R., 2015. ${ }^{13} \mathrm{C}$-metabolic flux analysis of co-cultures: A novel approach. Metab. Eng. 31, 132-139.

Ghosh, A., Nilmeier, J., Weaver, D., Adams, P. D., Keasling, J. D., Mukhopadhyay, A., Petzold, C. J., Martín, H. G., 2014. A peptide-based method for ${ }^{13} \mathrm{C}$ metabolic flux analysis in microbial communities. PLoS Comput. Biol. 10, e1003827.

He, L., Xiao, Y., Gebreselassie, N., Zhang, F., Antoniewicz, M. R., Tang, Y. J., Peng, L., 2014. Central metabolic responses to the overproduction of fatty acids in Escherichia coli based on ${ }^{13} \mathrm{C}$-metabolic flux analysis. Biotechnol. Bioeng. 111, 575-585.

He, W., Fu, L., Li, G., Jones, J. A., Linhardt, R. J., Koffas, M., 2015. Production of chondroitin in metabolically engineered E. coli. Metab. Eng. 27, 92-100.

Hollinshead, W., Rodriguez, S., Garcia-Martin, H., Wang, G., Baidoo, E., Sale, K. L., Keasling, J. D., Mukhopadhyay, A., Tang, Y., 2016. Examining Eschericihia coli glycolytic pathways, catabolite repression, and metabolite channeling using pfk mutants. Biotechnology for Biofuels. 
Ishii, N., Nakahigashi, K., Baba, T., Robert, M., Soga, T., Kanai, A., Hirasawa, T., Naba, M., Hirai, K., Hoque, A., 2007. Multiple high-throughput analyses monitor the response of $E$. coli to perturbations. Science. 316, 593-597.

Jones, J. A., Toparlak, Ö. D., Koffas, M. A., 2015a. Metabolic pathway balancing and its role in the production of biofuels and chemicals. Curr. Opin. Biotechnol. 33, 52-59.

Jones, J. A., Vernacchio, V. R., Lachance, D. M., Lebovich, M., Fu, L., Shirke, A. N., Schultz, V. L., Cress, B., Linhardt, R. J., Koffas, M. A., 2015b. ePathOptimize: a combinatorial approach for transcriptional balancing of metabolic pathways. Sci. Rep. 5, 11301.

Kayser, A., Weber, J., Hecht, V., Rinas, U., 2005. Metabolic flux analysis of Escherichia coli in glucose-limited continuous culture. I. Growth-rate-dependent metabolic efficiency at steady state. Microbiology. 151, 693-706.

Kind, S., Becker, J., Wittmann, C., 2013. Increased lysine production by flux coupling of the tricarboxylic acid cycle and the lysine biosynthetic pathway-Metabolic engineering of the availability of succinyl-CoA in Corynebacterium glutamicum. Metab. Eng. 15, 184195.

Leighty, R. W., Antoniewicz, M. R., 2011. Dynamic metabolic flux analysis (DMFA): a framework for determining fluxes at metabolic non-steady state. Metab. Eng. 13, 745-755.

Ma, F., Jazmin, L. J., Young, J. D., Allen, D. K., 2014. Isotopically nonstationary ${ }^{13} \mathrm{C}$ flux analysis of changes in Arabidopsis thaliana leaf metabolism due to high light acclimation. Proceedings of the National Academy of Sciences. 111, 16967-16972.

McClean, K. H., Winson, M. K., Fish, L., Taylor, A., Chhabra, S. R., Camara, M., Daykin, M., Lamb, J. H., Swift, S., Bycroft, B. W., 1997. Quorum sensing and Chromobacterium violaceum: exploitation of violacein production and inhibition for the detection of $\mathrm{N}$ acylhomoserine lactones. Microbiology. 143, 3703-3711.

Nakahigashi, K., Toya, Y., Ishii, N., Soga, T., Hasegawa, M., Watanabe, H., Takai, Y., Honma, M., Mori, H., Tomita, M., 2009. Systematic phenome analysis of Escherichia coli multiple-knockout mutants reveals hidden reactions in central carbon metabolism. Mol. Syst. Biol. 5, 306.

Nanchen, A., Fuhrer, T., Sauer, U., 2007. Determination of metabolic flux ratios from ${ }^{13} \mathrm{C}-$ experiments and gas chromatography-mass spectrometry data. Metabolomics: Methods and Protocols. 177-197.

Nargund, S., Misra, A., Zhang, X., Coleman, G. D., Sriram, G., 2014. Flux and reflux: metabolite reflux in plant suspension cells and its implications for isotope-assisted metabolic flux analysis. Mol. BioSyst. 10, 1496-1508.

Park, J. O., Rubin, S. A., Xu, Y.-F., Amador-Noguez, D., Fan, J., Shlomi, T., Rabinowitz, J. D., 2016. Metabolite concentrations, fluxes and free energies imply efficient enzyme usage. Nat. Chem. Biol.

Rühl, M., Hardt, W. D., Sauer, U., 2011. Subpopulation-specific metabolic pathway usage in mixed cultures as revealed by reporter protein-based ${ }^{13} \mathrm{C}$ analysis. Applied Environmental and Microbiology. 77, 1816-1821.

Rühl, M., Le Coq, D., Aymerich, S., Sauer, U., 2012. ${ }^{13}$ C-flux analysis reveals NADPHbalancing transhydrogenation cycles in stationary phase of nitrogen-starving Bacillus subtilis. J. Biol. Chem. 287, 27959-27970.

Sauer, U., 2006. Metabolic networks in motion: ${ }^{13} \mathrm{C}$-based flux analysis. Mol. Syst. Biol. 2.

Segre, D., Vitkup, D., Church, G. M., 2002. Analysis of optimality in natural and perturbed metabolic networks. Proc. Natl. Acad. Sci. U. S. A. 99, 15112-15117. 
Shearer, G., Lee, J. C., Koo, J. a., Kohl, D. H., 2005. Quantitative estimation of channeling from early glycolytic intermediates to $\mathrm{CO}_{2}$ in intact Escherichia coli. FEBS J. 272, 3260-3269.

Shlomi, T., Berkman, O., Ruppin, E., 2005. Regulatory on/off minimization of metabolic flux changes after genetic perturbations. Proc. Natl. Acad. Sci. U. S. A. 102, 7695-7700.

Stelling, J., Sauer, U., Szallasi, Z., Doyle, F. J., Doyle, J., 2004. Robustness of cellular functions. Cell. 118, 675-685.

Sumegi, B., Sherry, A. D., Malloy, C. R., 1990. Channeling of TCA cycle intermediates in cultured Saccharomyces cerevisiae. Biochemistry. 29, 9106-9110.

Sun, H., Zhao, D., Xiong, B., Zhang, C., Bi, C., 2016. Engineering Corynebacterium glutamicum for violacein hyper production. Microb Cell Fact. 15, 148-156.

Taymaz-Nikerel, H., De Mey, M., Ras, C., ten Pierick, A., Seifar, R. M., Van Dam, J. C., Heijnen, J. J., van Gulik, W. M., 2009. Development and application of a differential method for reliable metabolome analysis in Escherichia coli. Anal. Biochem. 386, 9-19.

Toya, Y., Ishii, N., Hirasawa, T., Naba, M., Hirai, K., Sugawara, K., Igarashi, S., Shimizu, K., Tomita, M., Soga, T., 2007. Direct measurement of isotopomer of intracellular metabolites using capillary electrophoresis time-of-flight mass spectrometry for efficient metabolic flux analysis. J. Chromatogr. A. 1159, 134-141.

van Heerden, J. H., Wortel, M. T., Bruggeman, F. J., Heijnen, J. J., Bollen, Y. J., Planqué, R., Hulshof, J., O'Toole, T. G., Wahl, S. A., Teusink, B., 2014. Lost in transition: start-up of glycolysis yields subpopulations of nongrowing cells. Science. 343, 1245114-1245122.

Wegman, J., Crawford, I. P., 1968. Tryptophan synthetic pathway and its regulation in Chromobacterium violaceum. J. Bacteriol. 95, 2325-2335.

Wheeldon, I., Minteer, S. D., Banta, S., Barton, S. C., Atanassov, P., Sigman, M., 2016. Substrate channelling as an approach to cascade reactions. Nature Chem. 8, 299-309.

Whitaker W. B., Jones J. A., Bennett R. K., Gonzalez J. E., Vernacchio V. R., Collins S. M., Palmer M. A., Schmidt S., Antoniewicz M. R., Koffas M. A. G., Papoutsakis E. T., 2016. Engineering the biological conversion of methanol to specialty chemicals in Escherichia coli. Metab. Eng. In Press.

Wiechert, W., 2001. ${ }^{13}$ C Metabolic Flux Analysis. Metab. Eng. 3, 195-206.

Wittmann, C., 2007. Fluxome analysis using GC-MS. Microb. Cell Fact. 6, 1.

Wu, G., Yan, Q., Jones, J. A., Tang, Y. J., Fong, S. S., Koffas, M. A., 2016. Metabolic Burden: Cornerstones in Synthetic Biology and Metabolic Engineering Applications. Trends Biotechnol. 34, 652-664.

Xiao, Y., Bowen, C. H., Liu, D., Zhang, F., 2016. Exploiting nongenetic cell-to-cell variation for enhanced biosynthesis. Nat Chem Biol. 12, 339-344.

Xiong, W., Morgan, J. A., Ungerer, J., Wang, B., Maness, P.-C., Yu, J., 2015. The plasticity of cyanobacterial metabolism supports direct $\mathrm{CO}_{2}$ conversion to ethylene. Nat. Plants. 1.

Xu, P., Bhan, N., Koffas, M. A., 2012a. Engineering plant metabolism into microbes: from systems biology to synthetic biology. Curr. Opin. Biotechnol. 24, 291-299.

Xu, P., Vansiri, A., Bhan, N., Koffas, M,A, 2012b. ePathBrick: a synthetic biology platform for engineering metabolic pathways in E.coli. ACS Synth. Biol. 1, 256-266

You, L., Berla, B., He, L., Pakrasi, H. B., Tang, Y. J., 2014. ${ }^{13}$ C-MFA delineates the photomixotrophic metabolism of Synechocystis sp. PCC 6803 under light- and carbonsufficient conditions. Biotechnol. J. 9, 684-692. 
Young, J. D., Shastri, A. A., Stephanopoulos, G., Morgan, J. A., 2011. Mapping photoautotrophic metabolism with isotopically nonstationary ${ }^{13} \mathrm{C}$ flux analysis. Metab. Eng. 13, 656-665.

Zamboni, N., Fendt, S.-M., Rühl, M., Sauer, U., 2009. ${ }^{13}$ C-based metabolic flux analysis. Nat. Protoc. 4, 878-892.

Zhao, S., Jones, J.A., Lachance, D.M., Bhan, N., Khalidi, O., Venkataraman, S., Wang, Z., Koffas, M.A.G., 2015. Improvement of catechin production in Escherichia coli through combinatorial metabolic engineering. Metab. Eng. 28, 43-53. 


\section{Figure legends}

Figure 1. Engineered $\boldsymbol{E}$. coli mutants for violacein production. (A) Graphical comparison of genetic constructs in strain E12 and Consensus. (B) Violacein biosynthesis pathway.

Figure 2. ${ }^{13} \mathrm{C}$-labeling experiment design and physiological performance of violaceinproducing $\boldsymbol{E}$. coli strains. (A): Graphical presentation of ${ }^{13} \mathrm{C}$ labeling experiment. (B): Sampling time points. Both engineered strains were grown in a minimal medium containing 15 $\mathrm{g} / \mathrm{L}\left[1,2-{ }^{13} \mathrm{C}\right]$, and samples were taken at five time points (i.e., $t_{1}$ to $t_{5}$ ), which represent five growth stages. (C): $\mathrm{OD}_{600}(\square)$ of the labeled culture and concentrations of glucose $(\mathrm{O})$, acetate $(\diamond)$, and violacein $(\triangle)$ at each time point.

Figure 3. Labeling patterns of free metabolites. (A) and (B) show the relative changes of measured labeling patterns (i.e., mass isotopomer distributions, MID) of free metabolite compared to the data at $t_{1}$ for strain Consensus and E12, respectively. (C) and (D) show the labeling patterns of ribulose 5-phosphate (Ru5P). (E) and (F) show the labeling patterns of glucose 6-phosphate (G6P). (G) and (H) show the labeling patterns of fructose 1,6-bisphosphate (FBP).

Figure 4. Metabolic flux phenotypes at different growth stages. (A) Heatmap representation of relative flux fold changes from $t_{1}$ to $t_{5}$. The relative changes were calculated based on the equation shown at the bottom of panel A with the fluxes at $t_{l}$ as the references. (B) Relative flux distribution around G6P branch node. Upper and lower numbers represent the relative fluxes of strain Consensus and E12, respectively. (C) Total relative flux (excluding exchange fluxes) in the central carbon metabolism. (D) Relative flux distributions of strain Consensus and E12 at $t_{4}$ and $t_{5}$. The flux values in Figure 4 are relative to the glucose uptake rate. Abbreviations for 
metabolites: 3PG, 3-phosphoglycerate; 6PG, 6-phosphogluconate; Ace, acetate; AceCoA, acetylCoA; AKG, alpha-ketoglutarate; CIT, citrate; DHAP, dihydroxyacetone phosphate; E4P, erythrose 4-phosphate; F6P, fructose 6-phosphate; FBP, fructose 1,6-bisphosphate; FUM, fumarate; G6P, glucose 6-phosphate; GAP, glyceraldehyde-3-phosphate; GLX, glyoxylate; ICIT, isocitrate; MAL, malate; OAA, oxaloacetate; PEP, phosphoenolpyruvate; PYR, pyruvate; R5P, ribose 5-phosphate; Ru5P, ribulose 5-phosphate; S7P, sedoheptulose-7-phosphate; SUC, succinate; SucCoA, succinyl-CoA; and X5P, xylulose 5-phosphate.

Figure 5. Evaluating the bottleneck of violacein production in strain Consensus. (A) The effects of tryptophan addition on the final violacein production at $t_{5}$. Error bars in (A) represent standard deviations from biological duplicates. (B) SDS-PAGE of soluble proteins in strain E12 and strain Consensus. The mutant strain with an empty plasmid containing no genes of violacein pathway was used as a negative control. The molecular weights of the five enzymes in violacein pathway: VioA, 49 kDa; VioB, 114 kDa; VioC, 49 kDa; VioD, 42 kDa; and VioE, 23 kDa. 
Figures

\section{Figure 1}
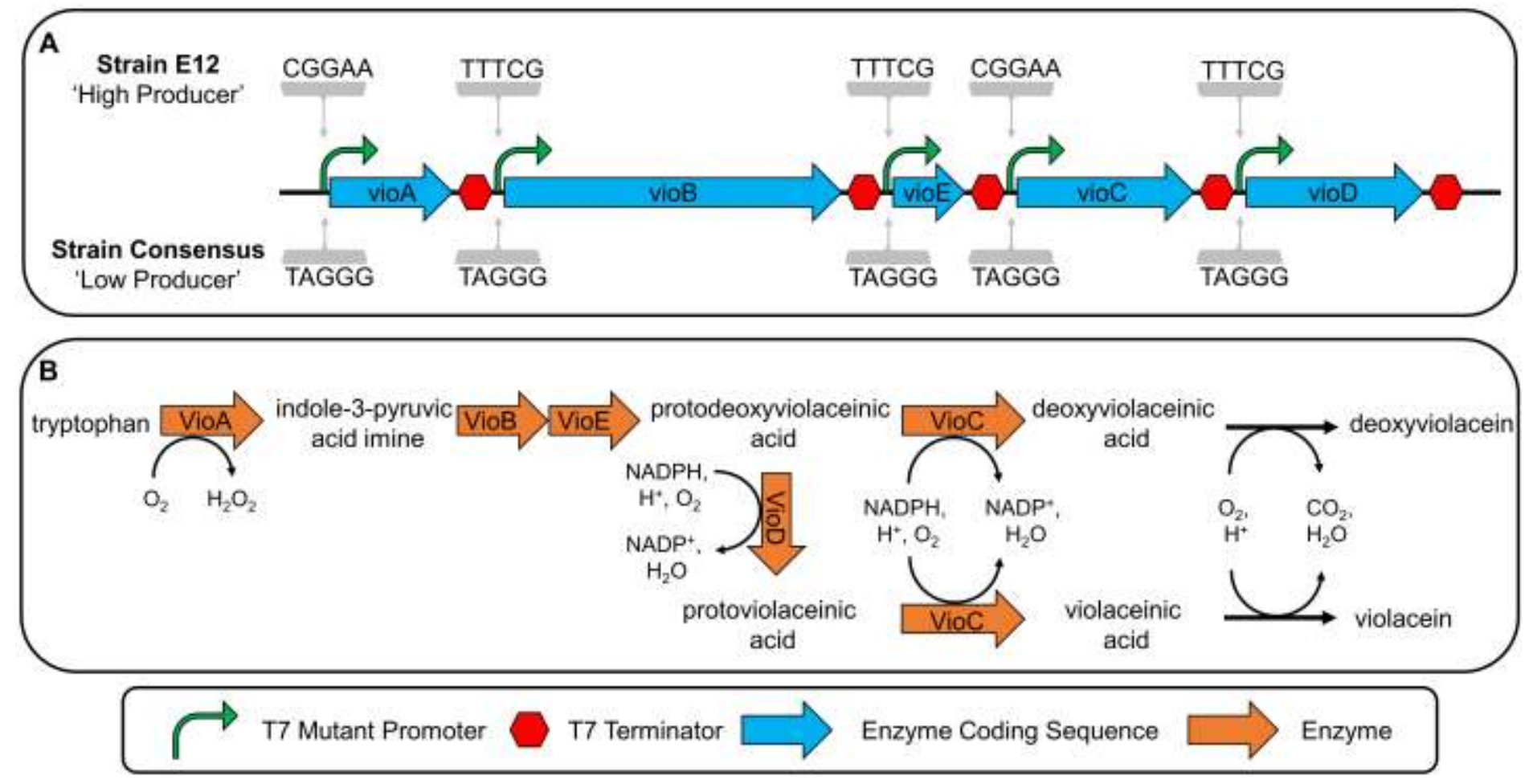
Figure 2
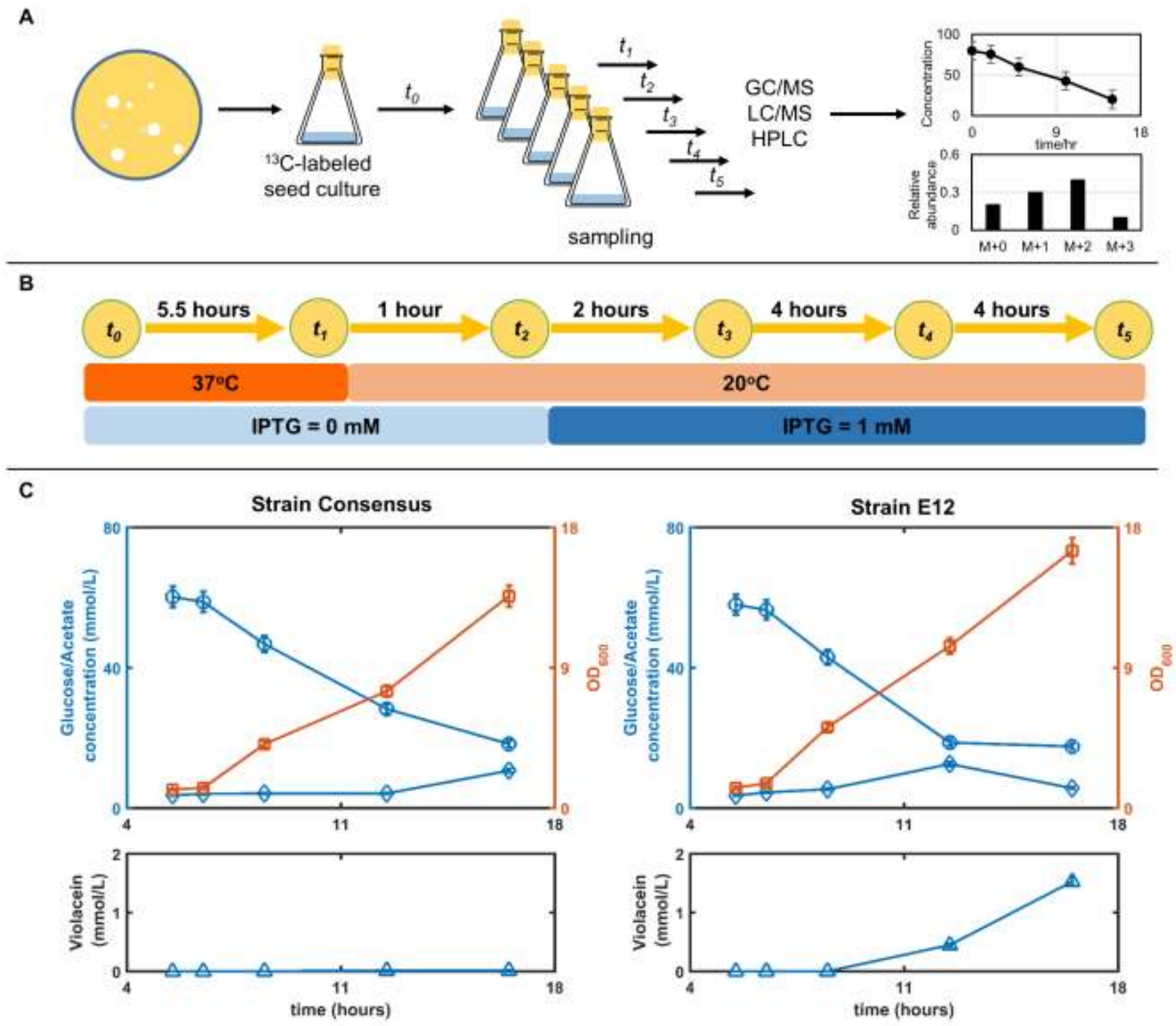
Figure 3
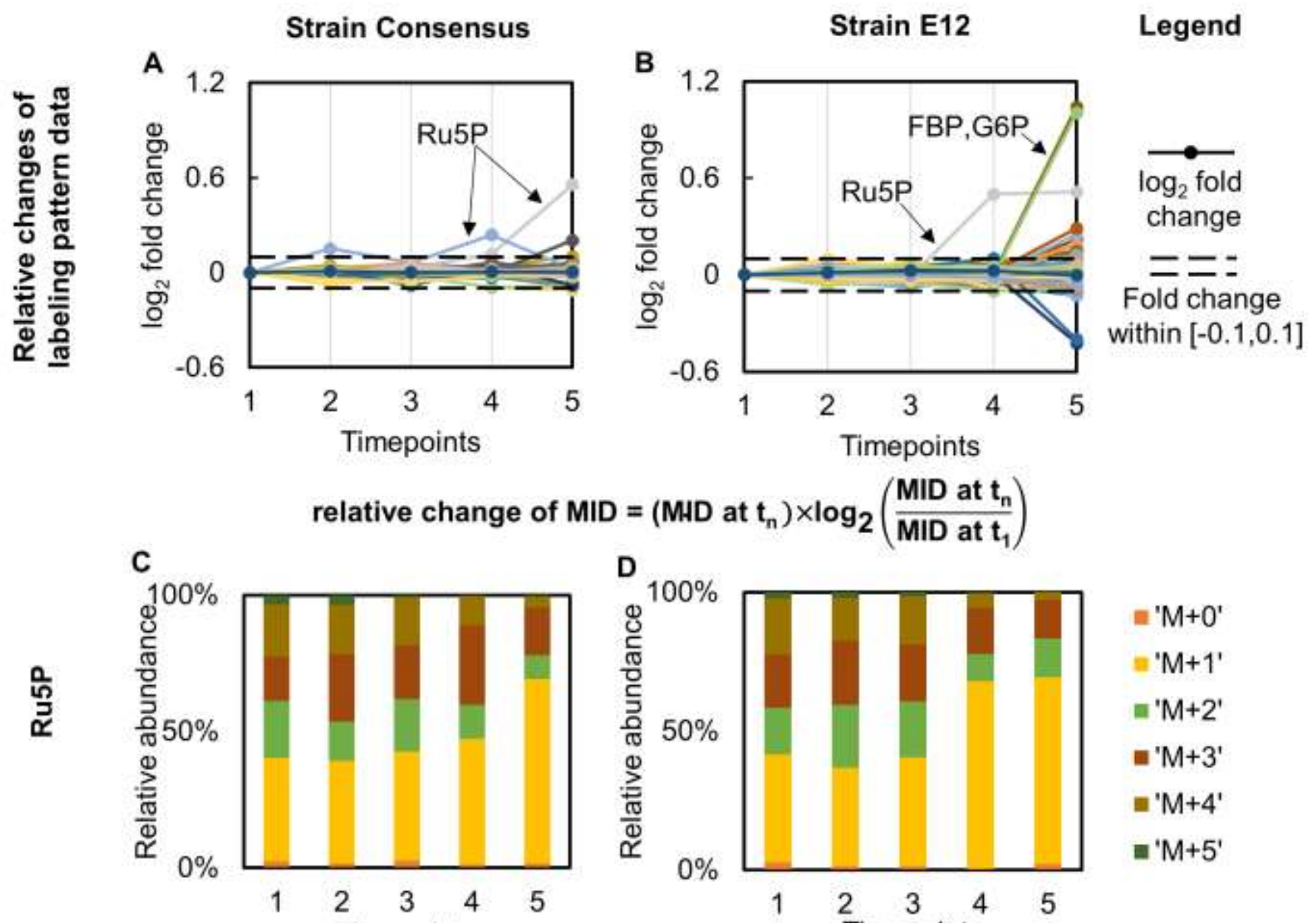

relative change of MID $=\left(\right.$ MHD at $\left.t_{n}\right) \times \log _{2}\left(\frac{\text { MID at } t_{n}}{\text { MID at } t_{1}}\right)$
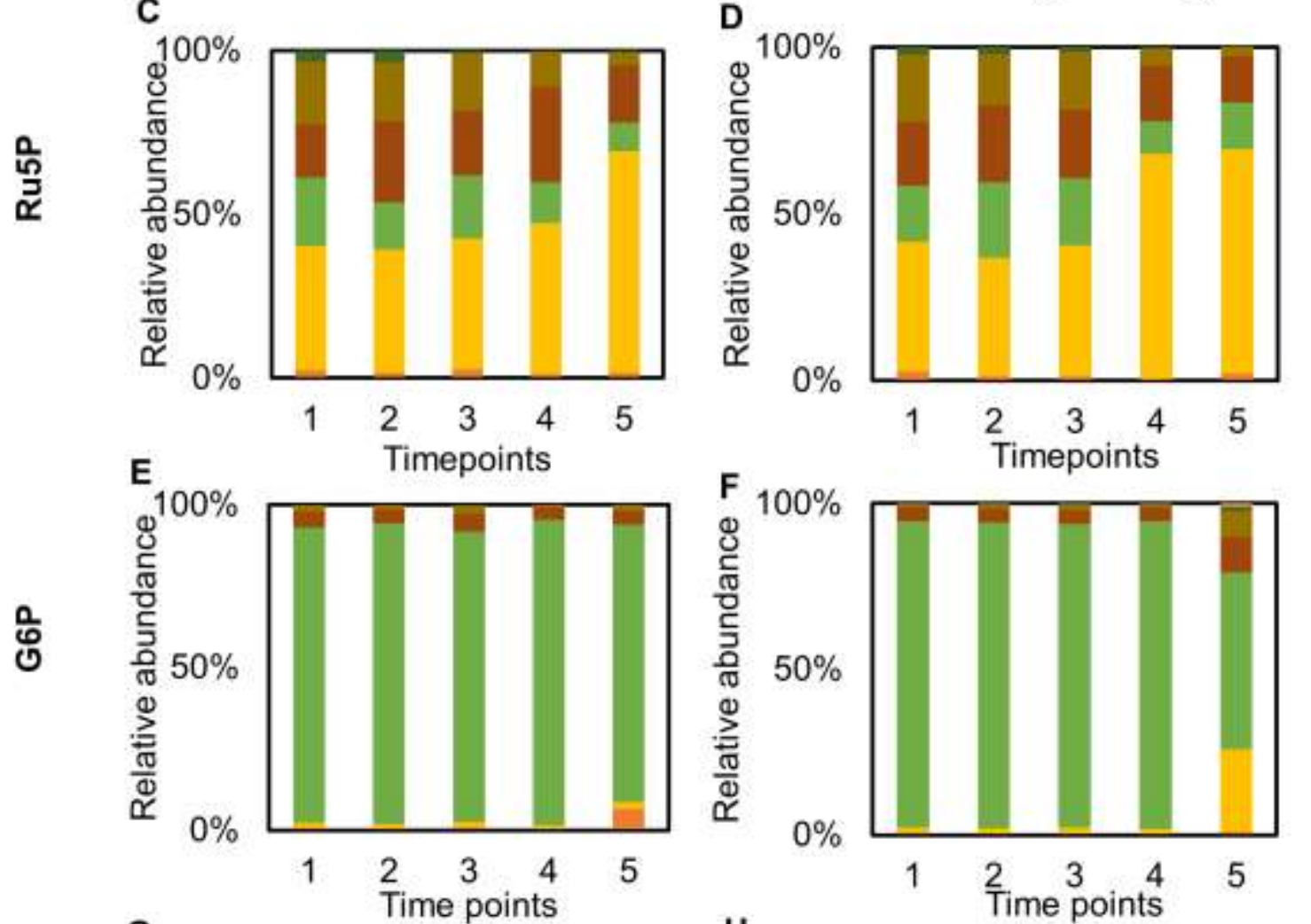

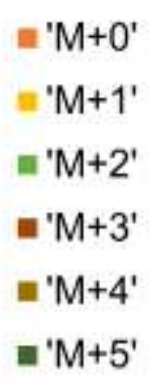
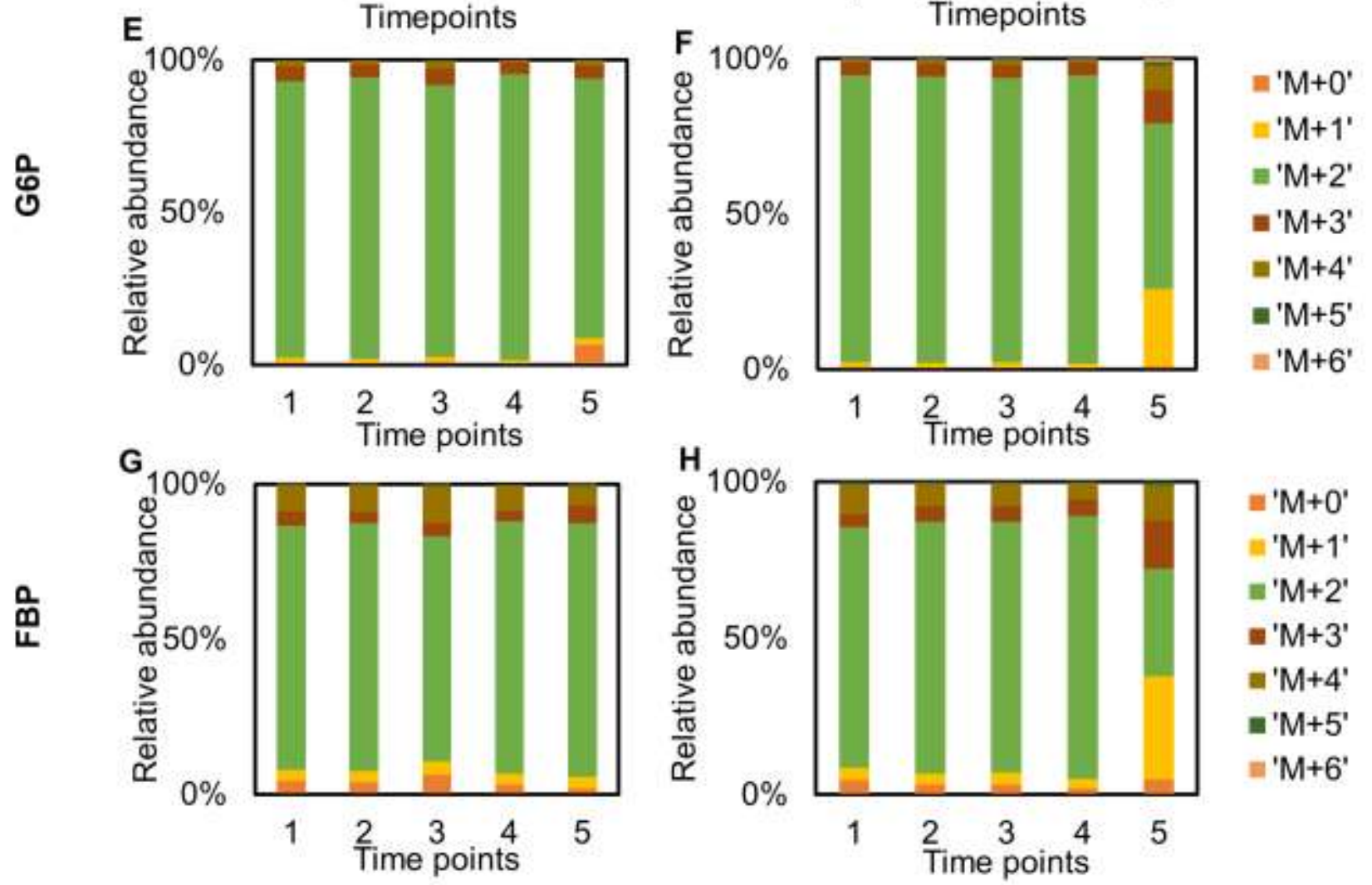
Figure 4

A

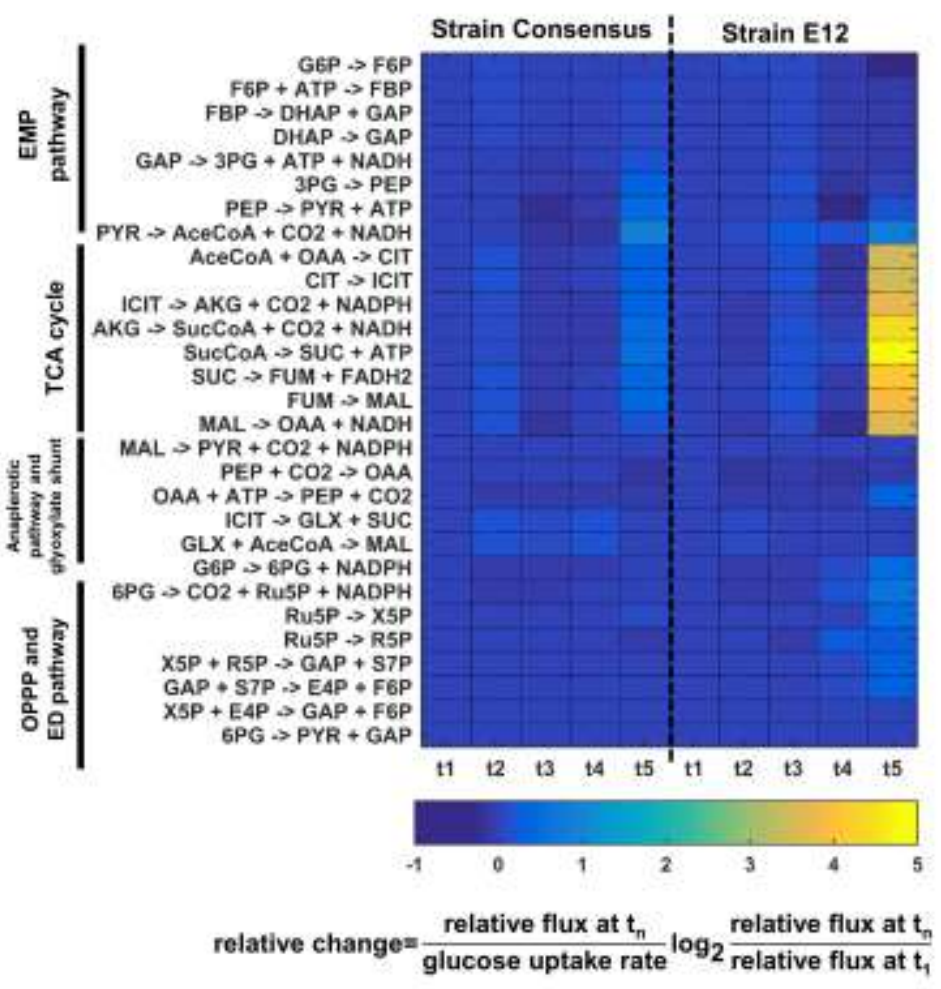

B

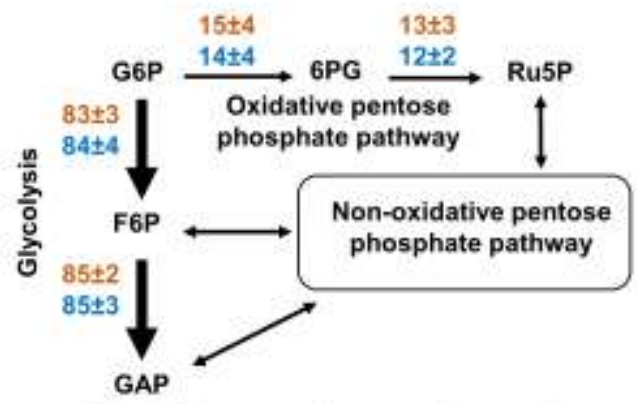

Strain Consensus (avg $\pm s t d v, t_{4}$ to $t_{s}$ ) Strain E12 (avg \pm stdv, $t_{1}$ to $t_{3}$ )

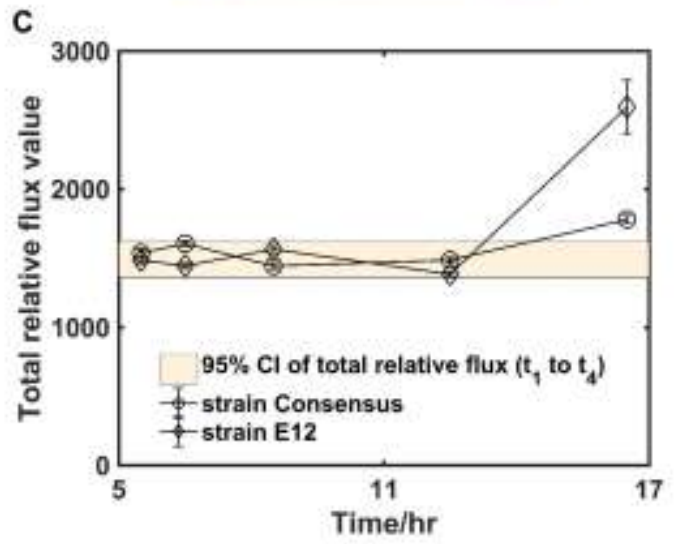

D

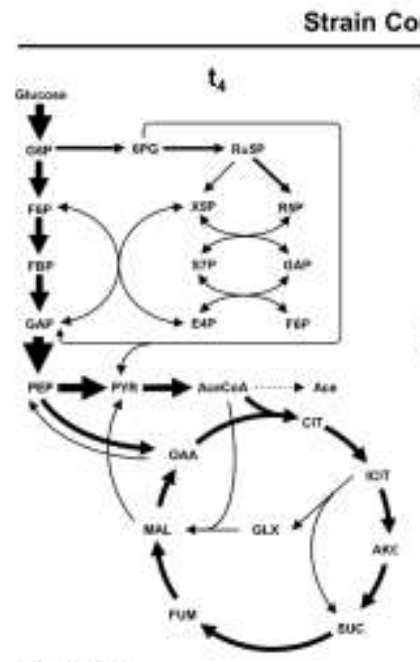

Biomass yield

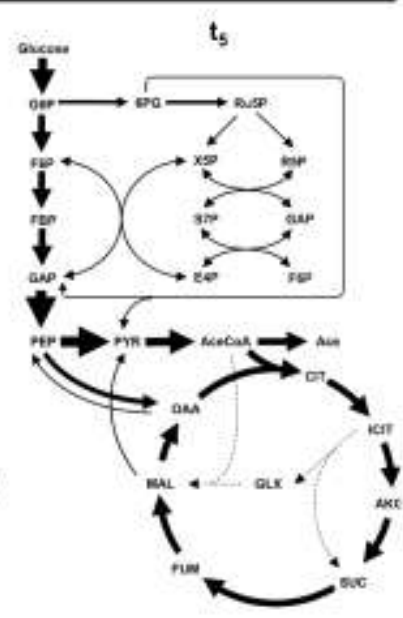

$4.2 \pm 0.3$

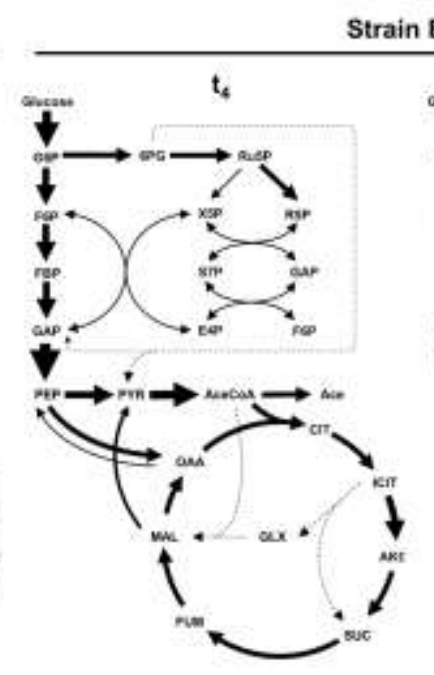

$3.6 \pm 0.4$ $t_{5}$

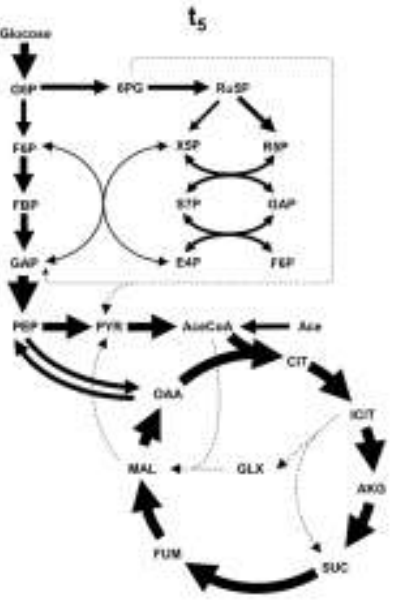

$0.1 \pm 0.3$

Legend 
Figure 5
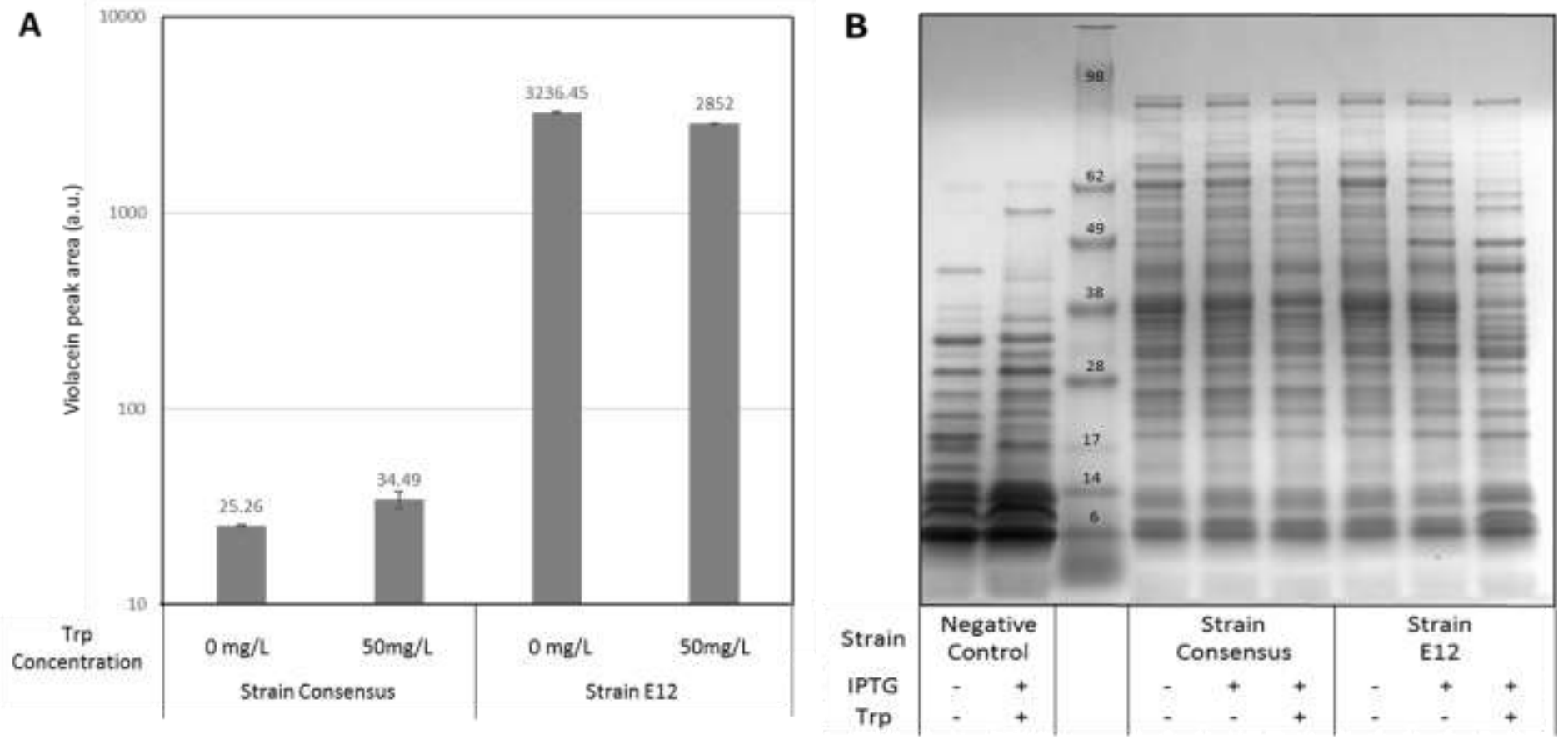